\title{
Criança com necessidades especiais de saúde: análise do cuidado prestado pela família
}

\author{
Child care with special health needs: Analysis of the care provided by the family \\ Niño com necessidades especiales de salud: Análisis del cuidado prestado por la familia. \\ Fernanda Lucas Lopes de Meirelles Leite ${ }^{1}$, Giovana Calcagno Gomes ${ }^{1}$, Alex Sandra Ávila Minasi ${ }^{1}$, \\ Camila Magroski Goulart Nobre ${ }^{1 *}$, Stella Minasi de Oliveira ${ }^{1}$, Daniel Gomes Severo'.
}

\section{RESUMO}

Objetivo: analisar o cuidado prestado pela família à criança com necessidades especiais de saúde. Método: Estudo qualitativo, realizado com 16 familiares de crianças egressas de uma Unidade Neonatal de município no sul do Brasil. Os dados foram coletados em 2016 por entrevistas semiestruturadas e submetidos à Análise Temática. Resultados: os resultados evidenciaram que o principal familiar cuidador foi a mãe. Referiram ter aprendido a cuidar na Unidade Neonatal; mobilizaram-se para o tratamento da criança com especialistas e exames; construíram a normalidade possível; transformaram o domicílio no principal contexto de cuidado, reestruturaram o cotidiano e uma rede de apoio social, abdicaram do trabalho, buscaram auxílio financeiro e recursos para o cuidado, vagas em creches e escolas e aprenderam a lidar com as tecnologias necessárias ao cuidado. Conclusões: é necessário investir no processo educativo da família para torná-la autônoma para o enfrentamento do processo de dependência de tecnologias pelas crianças.

Palavras-chave: Unidades de Terapia Intensiva Neonatal, Pessoas com necessidades especiais, Tecnologias em saúde.

\begin{abstract}
Objective: to analyze the care provided by the family to the child with special health needs. Method: A qualitative study was carried out with 16 relatives of children from a Neonatal Unit of a municipality in the south of Brazil. Data were collected in 2016 by semi-structured interviews and submitted to thematic analysis. Results: the results showed that the main family caregiver was the mother. They reported having learned to care in the Neonatal Unit; mobilized for the treatment of the child with specialists and examinations; constructed the normality possible; they transformed the home into the main context of care, restructured daily life and a social support network, abandoned their work, sought financial assistance and resources for care, places in day care centers and schools and learned to deal with the technologies needed for care. Conclusions: it is necessary to invest in the educational process of the family to make it autonomous to confront the process of dependence on technology by children.
\end{abstract}

Keywords: Neonatal Intensive Care Units, People with special needs, Health technologies.

\section{RESUMEN}

Objetivo: analizar el cuidado proporcionado por la familia al niño con necesidades especiales de salud. Método: Se realizó un estudio cualitativo con 16 familiares de niños de una Unidad de Neonatología de un municipio en el sur de Brasil. Los datos se recopilaron en 2016 mediante entrevistas semiestructuradas y se sometieron a análisis temático. Resultados: los resultados mostraron que la principal cuidadora familiar era la madre. Informaron haber aprendido a cuidar en la Unidad de Neonatología; movilizado para el tratamiento

1Universidade Federal do Rio Grande (FURG), Rio Grande/RS, Brasil.`E-mail: kamy_magroski@yahoo.com.br 
del niño con especialistas y exámenes; Construimos la normalidad posible; transformaron el hogar en el contexto principal de atención, reestructuraron la vida cotidiana y una red de apoyo social, abandonaron su trabajo, buscaron asistencia financiera y recursos para la atención, lugares en guarderías y escuelas y aprendieron a lidiar con las tecnologías necesarias para la atención. Conclusiones: es necesario invertir en el proceso educativo de la familia para que sea autónomo enfrentar el proceso de dependencia de las tecnologías por parte de los niños.

Palabras clave: Unidades de cuidados intensivos neonatales, personas con necesidades especiales, tecnologías de la salud.

\section{INTRODUÇÃO}

A Unidade de Terapia Intensiva Neonatal (UTIN) desempenha um importante papel para a vida dos recémnascidos ( $R N)$ que necessitam de cuidados especializados relacionados à urgências, emergências, bem como, a tratamentos clínicos e patológicos. Também é um espaço criado para receber RN, especialmente os prematuros, que são crianças fragilizadas pela própria prematuridade, que apresentem patologias variadas ou condições clínicas desfavoráveis à manutenção de sua vida no ambiente extrauterino. Este espaço hospitalar busca minimizar o impacto sofrido pelo RN, contribuindo com manutenção da vida. Desta forma, muitas crianças sobrevivem aos primeiros cuidados especializados disponibilizados dentro da UTIN, porém tornam-se dependentes de tecnologias que possibilitem sua qualidade de vida. Essas crianças são denominadas de Children With Special Health Care Needs no Brasil chamadas de Crianças com Necessidades Especiais de Saúde (CRIANES) (CARVALHO LDS e PEREIRA CDMC, 2017; TAVARES TS, et al.,2014).

O hospital torna-se um ambiente onde a presença, a participação e a interação dos pais e os profissionais de enfermagem ocorra de maneira efetiva, possibilitando o aprendizado e a aproximação com as tecnologias necessárias, para que o cuidado a CRIANES ocorra de maneira humanizada (BARRETO AP e INOUE KC, 2017; GARCIA FBG e EVANGELISTA IC, 2017).

Quando nos deparamos com crianças acometidas de doenças graves e crônicas, que podem interferir em suas demandas biológicas, psicológicas e até mesmo cognitivas, faz-nos pensar o quão grande e árduo tornase sua convivência com a necessidade de tratamentos medicamentosos, profissionais especializados e recursos tecnológicos, e ainda a incerteza da recuperação ou estabilização dos déficits e dificuldades provenientes da sua condição (TAVARES TS, et al., 2014).

Os recursos tecnológicos começam a fazer parte do cotidiano das CRIANES, sendo que essas demandas podem decorrer de doenças genéticas, doenças crônicas, malformações congênitas ou lesões decorrentes da prematuridade (GARCIA FBG e EVANGELISTA IC, 2017; LEMOS CJN e DITTZ ED, 2014).

Neste interim, faz-se necessário várias adaptações no cotidiano da família devido a necessidade da manipulação de tecnologias fundamentais para melhor o cuidado dessas crianças (REIS KMN, et al., 2017). O processo de adaptação a esta nova realidade, requer o desenvolvimento de habilidades para o cuidado. A família necessita de informações claras e objetivas da equipe multiprofissional, a fim de organizar-se para as novas demandas da CRIANES no domicílio, estabelecendo, desta forma, estratégias para seu cuidado (TAVARES TS, et al., 2014).

Neste período de adequação torna-se imprescindível que a enfermagem realize intervenções junto aos familiares da criança, sobretudo em períodos de internações, auxiliando e orientando sobre as demandas da doença, uso de tecnologias e insumos necessários para a integralidade do cuidado (TAVARES TS, et al., 2014; GARCIA FBG e EVANGELISTA IC, 2017).

Com o diagnóstico da necessidade especial da criança faz-se necessário a manipulação de tecnologias fundamentais para melhor cuidar a CRIANES (BARRETO AP e INOUE KC, 2017; GARCIA FBG e EVANGELISTA IC, 2017). 
Pelo exposto, tem-se como questão de pesquisa: quais os cuidados prestados pela família à criança com necessidades especiais de saúde? A partir dessa o estudo objetivou analisar o cuidado prestado pela família à criança com necessidades especiais de saúde. Acredita-se como relevante dar visibilidade à temática e favorecer a reflexão sobre a complexidade do cuidado familiar às CRIANES e a necessidade de atuação da enfermagem no suporte a essas famílias.

\section{MÉTODOS}

Trata-se de um estudo qualitativo, exploratório e descritivo, realizado com 16 familiares de crianças egressas de uma Unidade Neonatal de município no sul do Brasil. Participaram do estudo pais e mães de crianças egressas da UTIN que na alta hospitalar necessitavam de alguma tecnologia de cuidado e possuíam necessidades especiais de saúde. Como critério para a escolha dos participantes se utilizou: ser cuidador significativo da criança no domicílio, acompanhar a criança durante seu atendimento na instituição de saúde e ter 18 anos ou mais. Foram excluídos os familiares que cuidam eventualmente da criança. Os dados foram coletados entre março e julho de 2016 por meio de entrevistas semiestruturadas acerca do cuidado familiar à criança e de suas vivências com esse cuidado. Marcou-se dia e hora da entrevista, que ocorreu no domicílio ou no ambulatório do hospital. As entrevistas foram gravadas e transcritas para análise.

A análise dos dados deu-se pela técnica de Análise Temática operacionalizada em três etapas: Préanálise, na qual se realizou a leitura flutuante, o agrupamento das falas e elaboração das unidades de registro; Exploração do material, na qual os dados foram codificados; Agrupamento por semelhanças e diferenças; Organização das categorias; Tratamento dos resultados obtidos e Interpretação, na qual foram selecionadas as falas mais significativas para ilustrar a análise e realizar a busca de autores para dar suporte à análise (MINAYO M, 2011; TRIVINÕS ANS, 2009)

Os preceitos da resolução 466/2012 do Conselho Nacional de Ética em Saúde para a pesquisa com seres humanos do Brasil foram levados em consideração. O projeto foi submetido ao Comitê de Ética em Pesquisa na Área de Saúde - CEPAS e obteve parecer de aprovação N063/2014.

As falas dos participantes foram identificadas pela letra $\mathrm{F}$ seguida do número da entrevista, com vistas a garantir sua privacidade.

\section{RESULTADOS}

Participaram do estudo nove famílias de CRIANES egressas da UTIN, sendo respondentes dezesseis familiares, sendo nove mães e sete pais das CRIANES. Em todas, as mães, apresentou-se como a principal cuidadora. A renda familiar variou entre $R \$ 600,00$ e $R \$ 10.000,00$, sendo provida pelo pai (cinco famílias), pelo pai e pela mãe (três famílias) e por auxílio do governo (uma família).

As CRIANES possuíam entre dois meses e oito anos, sendo duas do sexo feminino e sete do sexo masculino. Apresentavam como necessidades especiais de saúde o uso de sonda nasoenteral; sonda de gastrostomia; cadeira de rodas; prótese auditiva; óculos; válvula de derivação peritoneal; órtese para sustentação dos membros inferiores; fraldas e concentradores de oxigênio de uso contínuo. Sete faziam uso contínuo de medicações. Todas nasceram prematuras. Os problemas apresentados pelas CRIANES que levaram a necessidade especial de saúde foram hidrocefalia, anóxia neonatal, displasia pulmonar, refluxo gastroesofágico, encefalopatia, Síndrome de Klinefelter e Síndrome de West.

\section{A qualificação da família para o cuidado especializado}

O período de internação da criança na UTIN foi utilizado pela família como estratégia de qualificação para o cuidado. Com a experiência cotidiana da hospitalização e apoio dos profissionais aprenderam a desenvolver o cuidado a CRIANES.

"O tempo dentro da UTIN me preparou para cuidar dele. Eu estava louca para sair da UTIN, mas ao mesmo tempo eu tinha medo de ir para casa e perder aquele auxílio, aquela força." (F2) 
"Claro que o aprendizado para cuidar em casa não foi suficiente. A gente sempre precisa de uma ajuda a mais, mas a ajuda dos profissionais da UTIN foi demais, foi o que me preparou para cuidar em casa." (F6)

"Se eu não tivesse passado aquele tempo no intermediário, no canguru, eu acho que não estaria preparada para ir para casa." (F7)

As necessidades especiais da criança impõem à família ter que conviver com tecnologias de cuidados, imperativas ao seu desenvolvimento. Nesse sentido, as famílias passam a lidar com cadeiras de rodas, alimentação por sondas de gastrostomia ou enteral, próteses e órteses, concentradores e torpedos de oxigênio, válvulas de derivação peritoneal, entre outras, com a finalidade de garantir uma qualidade de vida à criança.

"O neuro acredita que a desatenção possa ser da deficiência auditiva. Então agora com a prótese acredito que vá resolver. Estamos nos adaptando a ela e estimulando ele. Após o botox nas pernas e com as órteses, em dois meses ele começou a caminhar." (F5)

"Ele não caminha. Usamos ainda um carro tipo de bebê bem grande. Ainda não admito a cadeira de rodas. Estou tentado me convencer dessa ideia de que vai precisar de uma cadeira de rodas." (F14)

"Eu precisei me acostumar com a cor dele, com o barulho do concentrador de oxigênio, com o chiado no peito. Quando saio temos que levar o torpedo. Ainda estamos nos acostumando com esse aparato todo. A sonda ele arranca várias vezes ao dia, tenho que cuidar, saber se está no lugar correto. As gurias (enfermeiras da UTIN) revezavam-se e vinham aqui trocar a sonda." (F16)

"Ele toma remédios para a bronquite, ele tem displasia. Então tem as bombinhas, tem que dar os jatos, esperar para ele respirar dentro do espaçador, às vezes precisa nebulizar. Acabei comprando um nebulizador e um aspirador." (F2)

Conforme a família se qualifica e aprende a cuidar da criança, ela passa a enxergar o cuidado especializado como sendo natural ou normal. Essa estratégia de cuidado permite que a mesma desempenhe atividades básicas, na tentativa de observar progressos no desenvolvimento da CRIANES.

"Eu tento cuidar dele o mais dentro da normalidade possível. Eu tento deixar ele fazer as coisas, não ficar com essas coisas:_A ele é doente." (F3)

"Eu sei que ele tem um problema, mas eu tento agir o mais normal possível com ele." (F9)

"Se eu for ficar me preocupando, se eu for ver na internet tudo que acontece com essa síndrome eu vou pirar. Então eu levo para o lado normal." (F13)

\section{A transformação da família como estratégia de cuidado}

Com as necessidades da CRIANES as mães precisam conciliar a atividade laboral com o cuidado. Nesse sentido a família se reorganiza para auxiliar as principais cuidadoras. Essa estratégia faz-se necessário, pois em alguns casos, esta é a única fonte de renda da família.

"A minha irmã fica até às 16 horas e a outra irmã fica aos sábados." (F1)

"Meu horário de trabalho diminuiu muito. Pela manhã só trabalho até às 11:30 porque eu que faço comida e sou eu quem dá comida para ele. Às 13:30, 14:00 levo ele na escolinha e recomeço a atender às 14:30." (F5)

"Vou na leitaria, tiro o leite das vacas e volto para olhar ele. E assim passo os meus dias, para lá e para cá." (F4) 
Além de sua reorganização, algumas famílias ainda contam com o auxílio de uma babá para conseguir dar conta de todas as demandas de cuidado da criança.

"E para me ajudar nisso tudo eu sempre tive a babá dele." (F5)

"Ele tem uma babá que cuida dele desde que ele veio para casa. Ela está conosco até hoje. Então eu digo que ele tem duas mães, pois sem ela eu não conseguiria fazer tudo." (F14)

Há mães que se afastam da atividade laboral devido às demandas da criança, cuidando em tempo integral da mesma. No entanto, essa estratégia pode repercutir, gerando instabilidade econômica.

"Eu parei de trabalhar. Eu faço as coisas de casa com ela sempre na minha volta, ali no carrinho." (F6)

"Minha licença maternidade terminou antes mesmo dele vir para casa. Agora estou de licença interesse. Até ele completar uns dois anos não terei como trabalhar. Vou entrando com licenças, quando não der mais, terei que sair do trabalho." (F9)

Outra estratégia de cuidado utilizada pela família da CRIANES é a transformação do domicílio num contexto de cuidado. Observa-se a ampliação dos espaços de circulação da casa para melhorar 0 desenvolvimento das atividades cotidianas e potencializar o estímulo da criança.

"Eu mudei todos os móveis de lugar porque a casa era muito úmida. Eu tentava achar um lugar para colocar o berço que não pegasse a umidade da parede." (F2)

"As portas da casa são bem amplas para poder passar com a cadeira de rodas." (F12)

"Aqui em cima do consultório eu fiz uma sala que eu chamo de sala da estimulação. Ali tem tudo que ele precisa." (F5)

Após a alta hospitalar, é necessário fazer algumas adaptações da residência de forma a propiciar um bom desenvolvimento do cuidado e a confortabilidade da CRIANES. As famílias, em alguns casos, transformam a casa no mundo dela. Lá é o local onde tentam garantir sua estimulação e acessibilidade, inclusive realizando a fisioterapia no domicílio.

"Minha casa não precisou ser muito adaptada. A única coisa que fizemos foi mais um quarto, um quarto só para ele." (F11)

"Eu arrumei o quarto para ela, com todo conforto." (F10)

"Tudo que precisa para estimular eu compro. Aqui é o mundo dele, o mundo de acessibilidade." (F7)

"Na minha casa tem a sala da fisioterapia dele. Tem tudo, tudo que ele precisa." (F5)

Outra estratégia de cuidado é colocar a CRIANES no quarto dos pais. Assim a mãe cuida 24 horas por dia, observando a criança até mesmo enquanto ela dorme.

"Ele dorme comigo. Meu marido saiu do quarto e ele dorme comigo." (F4)

"Ele dorme comigo. Eu sempre fiquei muito agarrada com ele e muito ligada, muito preocupada. Tem que cuidar a respiração, a temperatura." (F5)

\section{Construindo e buscando estruturas de cuidado}

Para o desenvolvimento da saúde da CRIANES há a necessidade de insumos para o cuidado especializado. Destarte, outra estratégia da família é a busca de auxílio financeiro junto a Secretaria Municipal de Saúde, no próprio Hospital Universitário e na Promotoria Pública. As famílias acreditam que todo esse empenho se justifica na defesa dos seus direitos e para que ela seja bem cuidada e tratada. 
"Acho que foi esse cuidado, esse correr atrás que fez com que os médicos sempre investissem nele. A gente nunca desistiu. Não aguentava mais ficar na UTIN, foram seis meses. Então, eu disse quero ir embora com ele, não importa se for com oxigênio ou sonda para alimentação. Corremos na prefeitura, conseguimos o concentrador, os torpedos de oxigênio." (F9)

"Briguei com muita gente na UTIN por causa dele, para defender ele, os direitos de adquirir as coisas." (F4)

"Estou sempre correndo atrás de atendimento, vou atrás da enfermeira do posto para os encaminhamentos, vou na Secretaria de Saúde. A única coisa que não consegui ainda foi o passe livre do ônibus." (F8)

"Quando eu preciso de alguma coisa e não consigo aqui no hospital ou pela secretaria eu apelo. Agora tem o problema da fala, aqui não estou conseguindo a fono [fonoaudiologista], vou tentar na prefeitura e se precisar vou na promotoria." (F6)

A família passou a mobilizar-se para a realização do tratamento da CRIANE, buscando consultas com especialistas e a realização de exames, na tentativa de atender as necessidades e melhorar seu desempenho na realização das atividades de vida diária.

"Tudo que ele é capaz de fazer hoje é consequência do meu cuidado, da minha busca por respostas por tratamentos. Estamos fazendo os exames para poder colocar a prótese auditiva nele. Estamos investigando um pouquinho de deficiência visual. Em setembro fez botox nos membros inferiores, começou a usar a órtese." (F5)

"Eu sempre fiz estimulação com ele em casa. O fisioterapeuta dele é o mesmo desde que ele veio para casa." (F15)

Outra estratégia utilizada pelas mães, para cuidar, é a busca de vagas em creches e escolas, seja em classes especiais ou turmas regulares. Acreditam que essa seja uma maneira de seus filhos conviverem com outras crianças, outras pessoas, ter acesso à psicopedagoga e sala de recursos, sendo uma possibilidade de adquirir habilidades e socializar-se.

"Primeiro consegui uma vaga na creche da prefeitura, onde ele ficava meio período e convivia com outras crianças. Depois foi para a escola regular. Tem atendimento da psicopedagoga e vai na sala de recursos." (F3)

"Ele frequenta a escola normal, ensino regular." (F7)

"Então, antes de todos esses diagnósticos ele foi para a psicopedagoga e ela me orientou que era necessário colocar em uma escolinha para conviver com outras crianças, mas eu ficava com medo. Com o tempo fui criando coragem. Pela manhã ele fica com a babá e a tarde vai para a escolinha." (F5)

\section{DISCUSSÃO}

Com a ida para a casa com a CRIANES, a família assume a responsabilidade de executar as práticas de cuidados que podem variar dependendo da necessidade especial e das tecnologias utilizadas pela criança. Por ser uma CRIANES, a família lança mão de inúmeras estratégias de cuidado, necessárias para a manutenção da vida de seus filhos e enfrentamento das novas demandas (RAMOS, 2015; GARCIA FBG e EVANGELISTA IC.,2017).

Apesar do período de internação impactar a família ela o aproveita para se qualificar com vistas, a adquirir habilidades para cuidar. Estudo realizado em Unidade Pediátrica no Rio de Janeiro verificou-se a necessidade da família aprender no período de internação os cuidados que terá que realizar no domicílio de forma especializada. Esses novos saberes e habilidades são aprendidos com a enfermagem na prática social de 
cuidados das famílias e utilizados por estas como estratégias que favoreceram o desenvolvimento da CRIANES (GARCIA FBG e EVANGELISTA IC, 2017).

A internação na UTIN é um período em que o contato com os profissionais de saúde auxiliam no desenvolvimento de competências para o cuidado. Ao mesmo tempo em que a CRIANES recebe cuidados, a família aprende tanto observando, como recebendo orientações e realizando o cuidado de forma assistida. Em estudo realizado com familiares cuidadores de CRIANES em uma Unidade de Pediatria constatou-se que o ambiente hospitalar possibilita o exercício do cuidado à criança no processo de saúde-doença de forma integral e compartilhada entre família e profissionais de saúde, ampliando e qualificando o conhecimento dos familiares e os tornando adequados às necessidades das CRIANES (SOUSA FCP, et al., 2017).

Estudo que objetivou compreender a participação da família na segurança do paciente em unidades neonatais destacou a importância do esclarecimento aos pais sobre o estado do paciente, os cuidados oferecidos e equipamentos utilizados pelo filho na UTIN. Tais orientações devem ser realizadas com linguagem acessível e de maneira simples para a compreensão da família (SOUSA FCP, et al., 2017).

Verificou-se que o cuidado à CRIANES transcende as orientações recebidas pelos pais/familiares, pois as tecnologias para o seu desenvolvimento passam a fazer parte do seu cotidiano. A partir dessa transcendência podem perceber que o cuidado é normal, enxergando as potencialidades da criança e não as limitações. Nesse sentido, estudo realizado em hospital pediátrico de Fortaleza, destacou que para algumas famílias não houve estranhamento decorrente as deficiências ou dependências de tecnologias que repercutissem nas atividades diárias de seus filhos, compreendendo como normal o cuidado a ser dispensado e esses (FIGUEIREDO SV, et al., 2016).

O estudo apontou as mães como principais cuidadoras mostrando, assim, que a mulher permanece como responsável pelo cuidado dos filhos, sendo, frequentemente, quem assume o encargo com as necessidades da CRIANES (REIS KMN, et al., 2017; SILVA FHOB e CAVALCANTE LIC, 2015). Pesquisa que objetivou compreender e identificar a rede de apoio e rede social na qual as famílias estão inseridas, além de ratificar a genitora como sendo a principal cuidadora da CRIANES, também ressaltou a necessidade do auxílio de uma rede de apoio. Apontou que, às vezes, a mesma é frágil e restrita a alguns membros da família. No entanto, a mesma é imprescindível para o auxílio à cuidadora, reduzindo a sobrecarga advinda dos cuidados a seu filho (SIMONASSE MF e MORAES JMM, 2015).

As redes de apoio social se mostram essenciais na trajetória das famílias de CRIANES, uma vez que essas exigem, muitas vezes, cuidados em tempo integral. Frente a essa realidade, a família utiliza como estratégia o apoio social a fim de manter a atividade laboral, pois há uma demanda financeira alta devido às necessidades advindas dos medicamentos, equipamentos e adaptações (REIS KMN, et al.,2017)

Outra estratégia encontrada por algumas famílias é o auxílio de babás para assessorá-las nas demandas de cuidado de seus filhos. Pesquisa apontou que o auxílio da babá é importante para a assistência adequada para o cuidado e lazer das CRIANES. Esse apoio contribuiu para que algumas mães pudessem continuar sua atividade laboral (FIGUEIREDO SV, et al., 2016).

Porém, algumas mães acabam afastando-se da atividade laboral para se dedicarem exclusivamente ao cuidado de seus filhos. Fato também observado em estudo realizado em Minas Gerais com famílias de CRIANES, onde as demandas decorrentes da rotina de cuidado sobrecarregaram as mães. A necessidade de também cuidar da casa e de outros filhos, levaram a um contexto de exaustão, gerando prejuízos na qualidade de vida dessas mulheres (REIS KMN, et al., 2017).

Quando as mães se afastam do trabalho para estar junto de seus filhos que necessitam de atenção e cuidados especiais, acabam gerando para o núcleo familiar a redução de recursos oriundos da renda que auferiam. Ao mesmo tempo ocorre um aumento de gastos referentes às demandas de cuidado com as CRIANES (RAMOS LDC, et al.,,2015; FIGUEIREDO SV, et al., 2016).

Assim, muitas famílias recorrem ao auxílio do Estado. Estudo realizado em um município do interior do estado de São Paulo evidenciou a importância da assistência do governo através do Sistema Único de Saúde 
(SUS) que disponibiliza gratuitamente uma série de medicamentos e insumos, principalmente para as CRIANES. Porém, muitos medicamentos prescritos pelos médicos não são padronizados e ainda de alto custo, necessitando de requerimento formal para sua aquisição. Esse processo acarreta sentimentos de constrangimento e humilhação, pois é um procedimento moroso (OKIDO ACC., et al, 2016).

Em contrapartida, este mesmo estudo destaca como elementos facilitadores ao acesso dos medicamentos, o Benefício de Prestação Continuada (BPC), previsto em lei, que é equivalente ao valor de um salário mínimo nacional, o qual deve ser utilizado para o custeio do tratamento (BRASIL,2003). Salientase que o SUS garante atenção à saúde de forma integral, além da assistência terapêutica e a incorporação de tecnologias em saúde, assegurando a família e a criança acesso a medicamentos, exames, insumos, consultas com especialistas, fisioterapeutas, ou seja, todos os serviços e materiais necessários ao cuidado. Corroborando, o Estatuto da Criança e do Adolescente ratifica a garantia desses direitos, bem como, prevê o acesso à escola e creches com o intuito de melhorar o crescimento e desenvolvimento das CRIANES (BRASIL,2003; BRASIL,2010).

Apesar de ser previsto na legislação, existem barreiras e dificuldades levando a família a ter que buscar recursos legais para se valer do direito, esse fato pode levar ao seu desgaste. A enfermagem pode apoiar a CRIANES e sua família orientando-a acerca de seus direitos e dos caminhos para garanti-los (MELLO DF, et al., 2017).

Outra estratégia de cuidado referida pelas famílias das CRIANES é a realização de mudanças físicas no ambiente domiciliar de cuidado. A adaptação da estrutura física da residência visa atender as necessidades de cuidado a criança e dar mais conforto a mesma, possibilitando sua adaptação no domicílio, propiciandoIhe mais independência (RAMOS LDC, et al., 2015; REIS KMN, et al., 2017).

Este estudo apresenta como limitação o fato de ter sido realizado em um único contexto não possibilitando generalizações. Outros estudos devem ser realizados com vistas a verificar a atuação da enfermagem como parte da rede de apoio social das famílias tanto no hospital como no domicílio, potencializando suas estratégias para o cuidado às CRIANES.

\section{CONCLUSÃO}

Os dados do estudo permitiram concluir que o cuidado familiar à CRIANES dependente de tecnologia é complexo, exigindo da família a aquisição de novas habilidades e competências de cuidado. Destaca-se que é preciso repensar as práticas de cuidados da enfermagem para melhor subsidiar as famílias para o cuidado. É necessário melhorar os processos interativos entre enfermagem e familiares, investindo no seu processo educativo com o objetivo de torná-las autônomas, além de distinguir suas necessidades e demandas, favorecendo a diminuição das suas dificuldades para o enfrentamento do processo de dependência de tecnologias pelas CRIANES.

\section{REFERÊNCIAS}

1.BRASIL. Conselho Nacional de Secretários de Saúde. Legislação do SUS/Conselho Nacional de Secretários de Saúde. - Brasília: CONASS, 2003. 604 p.

2. BRASIL. Estatuto da criança e do adolescente. Lei n. 8.069, de 13 de julho de 1990 e legislação correlata [recurso eletrônico] 9aa ed. - Brasília: Câmara dos Deputados, Edições Câmara, 2010. 207 p.

3.BRASIL. Conselho Nacional de Secretários de Saúde. Legislação do SUS/Conselho Nacional de Secretários de Saúde.

- Brasília: CONASS, 2003. 604 p.

4.BRASIL. Estatuto da criança e do adolescente. Lei n. 8.069, de 13 de julho de 1990 e legislação correlata [recurso eletrônico] 9ª ed. - Brasília: Câmara dos Deputados, Edições Câmara, 2010. 207 p.

5.BARRETO AP, INOUE KC. Assistência humanizada em Unidade de Terapia Intensiva Neonatal (UTIN): a importância dos profissionais de enfermagem. Rev. Uningá Review. 2017; 15(1):66-71.

6.CARVALHO LDS, PEREIRA CDMC. As reações psicológicas dos pais frente à hospitalização do bebê prematuro na UTI neonatal. R. da SBPH. 2017; 20(2):101-22.

7.CHAGAS MCS, et al. Significado atribuído pela família ao cuidado da criança hospitalizada. av.enferm. 2017; 35(1):718. 
8.FIGUEIREDO SV, et al. Children with special health needs and family: implications for Nursing. Rev Bras Enferm. 2016; 69(1):79-85.

9.GARCIA FBG, EVANGELISTA IC. Discursos sobre cuidados na alta de crianças com necessidades especiais de saúde. Rev. Bras. Enferm. 2017, vol.70, n.1, pp.163-71.

10.LEMOS CJN, DITTZ ED. A organização familiar para o cuidado à criança em condição crônica, egressa da unidade de terapia intensiva neonatal. Texto Contexto Enferm, Florianópolis, 2014 Abr-Jun; 23(2):318-27.

11.MINAYO M. O desafio do conhecimento: pesquisa qualitativa em saúde. São Paulo: Hucitec; Rio de Janeiro: Abrasco, 2011.

12.MELLO DF, et al. Cuidar em enfermagem na primeira infância: contribuições do reconhecimento intersubjetivo. Rev. Bras. Enferm. 2017; 70(2):465-69.

13.OKIDO ACC, et al. Technology-dependent children and the demand for pharmaceutic al care. Rev Bras Enferm [Internet]. 2016; 69(4):671-7.

14.RAMOS LDC, et al. Maternal careat home for children with special needs. Invest. educ. enferm. 2015; 33(3):492-99.

15.REIS KMN, et al. A vivência da família no cuidado domiciliar à criança com necessidades especiais de saúde. Cienc. enferm. 2017; 23(1):45-55.

16.SILVA FHOB, CAVALCANTE LIC. Rotinas Familiares de Crianças com Necessidades Especiais em Família Adotiva. Psic. Teor. e Pesq. 2015; 31(2):173-80.

17.SIMONASSE MF, MORAES JMM. Crianças com necessidades especiais de saúde: impacto no cotidiano familiar. Rev. Pesqui. Cuid. Fundam. 2015; 7(3):2902-09.

18.SOUSA FCP, et al. Family participation in patient safety in neonatal units from the nursing perspective. Texto contexto - enferm. 2017; 26(3): e1180016.

19.TAVARES TS, et al. Caracterização do perfil das crianças egressas de unidade neonatal com condição crônica. R. Enferm. Cent. O. Min. 2014; 3(4):1322-35.

20.TRIVIÑOS ANS. Introdução à pesquisa em ciências sociais: a pesquisa qualitativa em educação. São Paulo: Atlas, 2009. 